\title{
A Relevance-Theoretic Study of Offensive Utterance in Film Dialogues
}

\author{
Yu Yang \\ Jilin Agricultural University \\ Changchun, China
}

\begin{abstract}
The present study probes into the nature of offensive utterance in film dialogues in terms of its generating mechanism and responding strategy. Methodologically, the study adopts the way of qualitative analysis and description of the data which are mainly extracted from classic film dialogues of China and the United States. Under the guidance of Sperber \& Wilson's relevance theory, this study generalizes the generating mechanism--- loss of ostension in the speaker's utterance and different cognitive environments between speaker and hearer; two major responding strategies ---conflicting response and moderating response. The research may be significant in providing guidelines for people on how to avoid offensive utterances to communicate smoothly. Besides, the screenwriters can be inspired to create more classic dialogues and thus to better achieve the communicative function of the film.
\end{abstract}

Keywords-film dialogues; offensive utterance; generating mechanism; responding strategy

\section{INTRODUCTION}

Offensive utterance is a kind of impolite language which exerts negative influences on interpersonal relationship. As a medium of cultural exchange, film dialogues could provide an illustration of the culture essence in films while conveying information and feeling. The success of a film cannot be separated from the impressive dialogues between different roles in the film. The forms of film dialogues are various, of which, offensive utterance plays an essential part in portraying a character, pushing the development of plot and disseminating national culture. The present study focuses on the analysis of the generating mechanism of offensive utterance and its responding strategy in film dialogues.

\section{LITERATURE REVIEW}

\section{A. Definition and function of offensive utterances in film dialogues}

The present study adopts Shen Zhiqi's definition of offensive utterance. In his article, Professor Shen interprets offensive utterance in his own way [10]. According to him, the speaker's speech consciously or unconsciously threats or derogates the hearer's personality, prestige, reputation, ideas, and interests. As a consequence, the hearer feels upset or offended. This type of speech is called "offensive utterance".

The offensive utterance in film dialogues is endowed with various functions: shaping a more vivid and lively character-offensive utterances could better exhibit the inner emotions and personalities of characters; revealing the identity and background of characters-- offensive utterances could directly reveal the occupation, educational background and social status of the roles so as to assist understanding the relationship between roles in film; strengthening the collision of characters-- offensive utterances sharpen the contradictions and promote development of the plot.

\section{B. Previous studies on offensive utterance}

Offensive language is proposed as a concept in the early twenty-first century. Initially, the scholars focus on the analysis of performance, consequences, and treatment of offensive language in a specific linguistic environment. Then a monograph is written by Jonathan Culpeper--- Impoliteness: using language to cause offence ${ }^{[2]}$. In his book, Culpeper expounds how to offend others with language, how to produce offensive language, the emotional consequences of the offending and the influence of the context on the offensive language. when studying offensive language, there are still a lot of specific analysis about the concrete offensive speech acts such as taboo and swearwords.

Basically, the previous studies of offensive utterances focus on the following aspects: definition and classification of offensive language; linguistic function of offensive language; how to produce offensive actions; how to counter verbal offence and how to interpret offensive language. The present study adopts Shen Zhiqi's definition of offensive utterance which is based on pragmatic interpretation.

Under the framework of relevance theory, this paper will further study the generating mechanism of offensive utterance in film dialogues and explore its responding strategy. According to relevance theory, communication is an ostensive-inferential process. The speaker conveys the intention to the hearer through the utterance. The hearer searches for optimal relevant contextual assumptions and infers the speaker's intention. In fact, inferential communication and ostension is one thing seen from two angles. From the communicator's point of view, it is ostension or making manifest his informative intention; and from that of the hearer it is inference, inferred from the evidence presented by the speaker. The important point of relevance theory provides a theoretical basis and a new perspective for the study of offensive utterances in the film dialogues. The theoretical framework of the present study may give insights into other pragmatic and communicative researches. 


\section{GENERATING MECHANISM OF OFFENSIVE UTTERANCE IN FILM DIALOGUES}

Offensive utterance in the film dialogues is the microcosm of the conflicts between people. Different roles in the film will also assume different communicative roles in different contexts. The contradictions between different characters lead to their inner psychological conflict. When dialogue takes place, it is necessary to involve the psychological conflict in the speeches, which can lead to the outbreak of offensive utterance. Based on the categorization of the data collected, the generating mechanism mainly falls into two aspects: loss of ostension in the speaker's utterance and different cognitive environments between speaker and hearer

\section{Loss of ostension in the speaker's utterance}

Mutual-manifest between the communicators is the basis for achieving relevance. When people communicate with each other, the speaker should provide an ostensive stimulus so that the hearer can infer his intended meaning. Once relevance is established, communication may be carried out smoothly. In film dialogues, the loss of ostension in speaker's speech could generate offensive utterance. The loss of ostension in speaker's utterance is realized in three ways: excessive or insufficient information, ambiguity and indirectness in speaker's utterances.

1) Excessive or insufficient information in speaker's utterances

In communication, the speaker usually provides exact information to the hearer. But in some cases, speaker often omits or adds more information in their utterances. Such elliptical or redundant information the speaker provides may offend the hearer. Consider the following dialogues in the film Big Shot's Funeral:

Lucy: What's the meaning of "state"?

Yoyo: Ideological consciousness.

Lucy: To be concrete.

Yoyo: You can see as far as ten inches; I can see as far as one hundred inches; Taylor can see as far as one thousand inches; Buddha can see infinitely far. Therefore, we are in different spiritual state. Buddha has long term vision and you are short-sighted.

In this dialogue, Lucy requests Yoyo to give further illustration of "the state". Yoyo provides more information than what she needs. Some sentences are irrelevant to the question. Yoyo ends his explanation with ironic words "shortsighted". The excessive information in Yoyo's utterances results in the loss of ostension in communication, which offends the hearer Lucy.

\section{2) Ambiguity in speaker's utterances}

Ambiguity is a type of uncertainty of meaning in which several interpretations are plausible. Usually, a sentence has more than one meaning in different contexts, which could lead to the loss of ostension in communication. Look at the following dialogues in film A Chinese Odyssey:

Ba Jie: I have been outrivaled him in the wisdom and kung fu, but now I'm afraid he has advantage over me because he has another assistant Zixia.
Sha Seng: But you have me.

Ba Jie: Just because of you, we will be defeated totally by them.

In the above conversation, Ba Jie's words "but now I'm afraid he has advantage over me because he has another assistant Zixia." make himself misunderstood by Sha Seng. Sha Seng takes it for granted that $\mathrm{Ba}$ Jie is overwhelmed by the enemy. He inferred $\mathrm{Ba}$ Jie's real intention by the reply "Just because of you, we will be defeated totally by them." Ba Jie's ambiguous utterances lead to the loss of ostention to the hearer Sha Seng. After understanding Ba Jie's message, Sha Seng feels shameful and offended.

\section{3) Indirectness in speaker's utterances}

When people communicate, they sometimes express their ideas indirectly. In other words, the speaker intends the hearer to infer his real meaning depending on the specific context. Such indirectness can easily offend the hearer. The following example falls into this category:

Julie: I think the tree looks particularly beautiful in this light. Don't you?

Bryce : If by "beautiful" you mean "unbelievably ugly" then, yes, I would agree.

\section{--- Flipped}

In this dialogue, Julie likes a fig tree very much, so she tells Bryce that the tree was so beautiful in the sun. But Bryce doesn't appreciate the tree. He deliberately misinterprets the word "beautiful" as "unbelievably ugly" in his reply so as to deny Julie's opinion. Bryce's indirect speech infringes Julie's face, which leads to the conflict.

\section{Different cognitive environments between speaker and hearer}

"A cognitive environment of an individual is a set of facts that are manifest to him". (Sperber \& Wilson, 1995) It consists of not only all the facts that he is aware of, but also all the facts that he is capable of becoming aware of, in his physical environment. The information in the cognitive environment is composed of three aspects: logical, encyclopedic and lexical information. In film dialogues, the different cognitive environments between speaker's and hearer's will directly lead to the production of offensive speech, which mainly divided into three types: different cognitive categories, different personal values and different social roles

\section{4) Different cognitive categories}

Lu Dahai: You drowned two thousand and two hundred workers on purpose and deducted three hundred dollars for each life lost! You will die without descendants.

Zhou Puyuan: ---

\section{---Thunderstorm}

In the film "Thunderstorm", Lu Dahai is Zhou Puyuan's son, but Lu Dahai is hidden from the fact and he doesn't know Zhou Puyuan is his father. Lu Dahai and Zhou Puyuan have different cognitive categories. "You will die without descendants" in Lu Dahai's speech harms his father unconsciously and constitutes offensive utterances

5) Different personal values 
Jia (to Yi): You shouldn't marry with a person from rural places. People there are mean and troublesome. When you get married, many relatives you don't know will come to you for help.

$$
\text { Bing (very angrily) : Are the city people all good? }
$$

In the film "Feng Shui ", Jia, Yi and Bing are roommates. One day, Jia and Yi are talking and Bing is reading. Jia and Yi live in city and ignore the fact that Bing comes from countryside. "Mean and troublesome" in their remarks about rural people makes Bing misunderstand it as insults and disrespect. The offence is generated easily because of different personal values.

\section{6) Different social roles}

Driver (to passenger) : The seat is reserved for the old, sick and pregnant people. Stand up and give way to the good ones.

$$
\text { ---Feng Shui }
$$

On a crowded bus, a pregnant woman struggled to grab a handrail, and a man in front of him did not see. The driver requests the man to offer his seat to the pregnant woman. The driver and the passenger assume different social roles. The driver considers that the seat is offered to the special group of people. But his utterances offend the man by the meaning "he is not kind" and undermine his reputation. In this example, the different social role is the cause for production of offensive utterances.

In this section, we have discussed the generating mechanism of offensive utterance in film dialogues. The production of offensive utterance is the result of interaction between speaker and hearer. Understanding the generating mechanism is helpful for people to avoid offensive behavior and choose a favorable responding strategy.

\section{RESPONDING STRATEGY TO OFFENSIVE UTTERANCE}

Offensive utterance in film dialogues is a kind of impolite phenomenon in the communication. It will threaten the hearer's face and infringe upon his rights. Therefore, the hearer may adopt different responding strategies accordingly. Two strategies have been found through the analysis of the data.

\section{A. Conflicting response}

Conflicting response is a type of response by collision. In order to maintain his face and dignity, the hearer opposes and counter attacks the speaker's words to express his dissatisfaction in tough and uncooperative attitude. Conflicting responses can be often achieved with threats and irony. Consider the following dialogue:

Nike: I can only adopt this stupid method in face of a fool like you.

Judy: Say it again, you are dead.

\section{---Contract Lover}

Nike hurts Judy by the words "stupid" and "fool" deliberately. In order to maintain his face and self-esteem, Judy responds his remarks with "you are dead". Judy vents her anger in the way of threatening and warning. This is a typical conflicting response.
Cui Ping: Men are doomed to meet the pretty minxes who are flirty and raunchy.

Wan Qiu: My sister, if a woman is stupid and not attractive, her husband would have an affair.

$$
\text { ---Lurk }
$$

In the dialogue, Cui Ping expresses her hidden jealousy and hatred by means of words "minxes", "flirty" and "raunchy". These offensive utterances insult Wan Qiu terribly. She responds with "a woman is stupid and not attractive" to imply that Cui Ping is foolish and stupid. By using irony, Wan Qiu saves her face while conducting a powerful attacking response to Cui Ping.

\section{B. Moderating response}

Moderating response is a skilful response. while maintaining his face and dignity, the hearer also gives considerations to the speaker's self-esteem to avoid a direct collision. The hearer expresses his dissatisfaction in mild and tactful style. Moderating response is an indirect language attacking. It is usually achieved by rhetorical questions or deliberate misinterpretations. Consider the following dialogues in the film Fortress Besieged:

Zhao Xinmei: What did you learn abroad?

Fang Hongjian: Philosophy.

Zhao Xinmei: From the viewpoint of people majored in science, learning philosophy is exactly the same with learning nothing.

Fang Hongjian: Mr Zhao, you'd better go to see oculist. There must be something wrong with your eyesight if you look at things in this way.

Zhao offends Fang by saying that "learning philosophy is exactly the same with learning nothing." Fang doesn't conduct language attack directly. Instead, he makes use of the polysemy of "eyesight" to deliberately misinterpret it as "ability to see". "Something wrong with your eyesight" in Fang's response is indicating Zhao's opinion is ridiculous. The invisible linguistic attack doesn't result in direct collision. Fang expresses his dissatisfaction without infringing on Zhao's face.

Miss Su: what a poser? I called you last night, and you didn't call me back today. You wouldn't come until I invite you.

Miss Tang: Do I deserve a poser? I follow the request of others. Even if we are invited to come, so what? If I have not been here, it is actually playing the card.

Miss Su called Miss Tang the day before to find out whether Tang had a date with Fang instead of greeting her. Therefore, Miss Su's "what a poser!" is actually a reproach on Miss Tang. In order not to destroy the relationship, Miss Tang immediately responds by rhetorical questions "Do I deserve a poser? We obey the request of others. Even if we are invited to come, so what?" to maintain each other's self-esteem, at the same time, she expresses her dissatisfaction with Miss Su's accusation indirectly.

Conflicting responding strategy can be more effective to maintain the face and dignity of the hearer, but it will lead to collision. Moderating responding strategy seems to be more 
compromised, but it can be employed to achieve harmony of communication. Offensive utterance frequently occurs in verbal communication. Because of the different backgrounds and cognitive environments, the hearers could adopt different responding strategies according to various circumstances.

\section{CONCLUSION}

Film is one vehicle for cultural communication and film dialogue is one of the major means to interpret the cultural connotation in the film. The present study investigates offensive utterance in film dialogues from two dimensions: generating mechanism and responding strategy. For each perspective, this paper is mainly a qualitative description and analysis of the data under the guidance of some concepts of Relevance Theory, such as cognitive environment, ostensiveinferential communication, and context and so on. The data used in this paper are mainly extracted from classic films in China and other countries.

The major findings in this study are: the generating mechanism--- loss of ostension in the speaker's utterance and different cognitive environments between speaker and hearer; responding strategy to offensive utterances: conflicting response and moderating response.

The findings of this research may shed lights on other linguistic researches. Theoretically, this research has made further efforts to support the view on human cognition and communication. At the same time, the film dialogue is a classic application of language in social interactions. It can promote the study of pragmatic theory from multi-perspectives, and it is easier for people to understand the pragmatic theories. Practically, the research may be significant in directing our daily communications. Offensive utterances could be avoided through understanding its generating mechanism. Once it occurs, the proper strategies can be employed to communicate smoothly. Besides, the screenwriters can be inspired to create more classic dialogues and thus to better achieve the communicative function of the film.

\section{REFERENCES}

[1] Brown, P. \& S. C. Levinson Universals in Language Usage: Politeness Phenomena. In E. Goody(ed.) Questions and Politeness: Strategies in Social Interaction. Cambridge: Cambridge University Press, 1978.

[2] Culpeper, Jonathan. Towards an anatomy of impoliteness[J].Journal of Pragmatics, 1996, (25) : 349-367.

[3] Culpeper, Jonathan. Impoliteness: Using Language to Cause Offence [M]. London: Cambridge University Press, 2009.

[4] Derek Bousfield. Impoliteness in Interaction[M].Amsterdam: John Benjamins Publishing Company.2008

[5] Feinberg, Joel. Offense to Others (The Moral Limits of the Criminal Law, Volume Two [M].Oxford University Press, 1985.

[6] He Ziran. A Study of Pragmatics. [M]. ChangSha: Hunan Educational Press.2006.

[7] Kaye, Barbara. \& Barry Sapolsky. Offensive Langauge in Prime Time Television: Before and After Content Ratings Journal of Broadcasting \& Eletronic Media 2001(2): 303-319

[8] Leech, G. Principles of Pragmatics [M]. London: Longman, 1983.

[9] RanYongping. A Pragmatic Analysis of Deliberate Offensive Utterances in Interpersonal Conflicts [J]. Journal of Foreign Language, 2011, (3).

[10] Shen Zhiq. The Pragmatic Analysis of Offensive Speech Act in Chinese [J].TC SOL Studies, 2010 (2).

[11] Shoemaker David. W. Dirty Words and the Offensive Principle .Law and Philosophy[J], 2000, Vol.19,No.6:545-584.

[12] Sperber,D \& Wilson, D. Relevance:Communication and cognition [M]. Oxford: Blackwell.1986/1995.

[13] Sperber,D \& Wilson,D. Irony and relevance: A reply to Seto,Hamamoto and Yamanashi[J]. In: Robyn Carston and Seji Uchida (eds.),Theory: Applications and implications:283-293. Amsterdam: John Benjamins. 1998. 